\title{
FAST COMMUNICATION
}

\section{A CONSTRAINED STRING METHOD AND ITS NUMERICAL ANALYSIS*}

\author{
QIANG DUं AND LEI ZHANG Z $^{\dagger}$
}

\begin{abstract}
A constrained string method is developed to solve the saddle-point problem with constraints. Based on the intrinsic description of the string method, Lagrange multipliers are employed for treating the constraints. Various mathematical properties are established such as the conservation of the constraints and the energy dissipation law. We also investigate time discretization schemes for the constrained string method and discuss possible alternative ways to enforce the constraints. Some numerical examples are presented for illustration.
\end{abstract}

Key words. Constrained string method, minimum energy path, saddle point, constrained optimization, Lagrange multiplier, augmented Lagrangian.

AMS subject classifications. 65K10, 65M12, 65Z05.

\section{Introduction}

The computation of saddle points and minimum energy paths has been of much interest in many areas of application, such as conformational changes in macromolecules, chemical reactions, diffusion in condensed-matter systems, and nucleation events during phase transformations $[16,17,18]$. Several numerical methods have been developed for computing the MEPs, including the Nudged Elastic Band (NEB) method $[10,11]$ and more recently the string method and its various improvements $[5,6]$. The relevant saddle point can be identified with the highest energy point on the MEP [13].

In practical applications, the computation of MEPs and/or saddle points may be subject to one or more constraints. In this brief report, we adopt the idea of the string method and the method of Langrange multipliers to develop a constrained string method for finding the MEPs and saddle points subject to general constraints. While the resulting method is very natural and straightforward, we discuss some interesting mathematical properties, not all of which are obvious. For instance, we not only establish the energy dissipation law and the conservation of constraints, but also show that the Lagrange multipliers may be taken to be independent of the string parametrization. Furthermore, we examine other alternatives for dealing with constraints including both the penalty method and the augmented Lagrangian method. We also conduct numerical experiments to complement our derivation and analysis and to reveal the potential applications of the constrained string method.

The rest of the paper is organized as follows: a brief review of string method is presented in the section 2 . In section 3 , we describe the mathematical formulation of the constrained string method along with detailed analysis. In section 4, we present the time discretization of the constrained string method. In section 5 , the numerical

${ }^{*}$ Received: November 20, 2008; accepted (in revised version): February 17, 2009. Communicated by Jack Xin.

This research is supported in part by the NSF grant DMS-0712744 and NSF IIP-541674 Center for Computational Materials Design (CCMD).

${ }^{\dagger}$ Department of Mathematics and Department of Materials Science and Engineering, Pennsylvania State University, PA 16802, USA (qdu@math.psu.edu). http://www.math.psu.edu

${ }_{\ddagger}$ Department of Mathematics, Pennsylvania State University, PA 16802, USA. Present address: Department of Mathematics, University of California, Irvine, CA 92697, USA (zhangl4@uci.edu). 
algorithm is described for the constrained canonical string method and we use a 3D example to illustrate the developed method. In section 6 , we discuss possible alternatives to treat the constraints, including the penalty formulation and the augmented Lagrangian method. Final conclusions are made in section 7 .

\section{The string method and its variants}

The string method was first developed by E, Ren, and Vanden-Eijnden [5] for computing the minimum energy path (MEP) and saddle points for barrier-crossing events. The method proceeds by evolving strings, i.e., smooth curves with intrinsic parametrization, to the most probable transition path between two metastable regions in the configuration space. More specifically, given a potential energy $E=E(x)$ with at least two local minima, at $a$ and $b$, a MEP refers to a smooth curve $\varphi^{*}$ connecting $a$ and $b$ that satisfies

$$
(\nabla E)^{\perp}\left(\varphi^{*}\right)=0
$$

where $\nabla E(\varphi)^{\perp}$ is the component of $\nabla E(\varphi)$ normal to a curve $\varphi$. That is, let $\hat{\tau}$ be the unit tangent of the curve $\varphi$, then

$$
(\nabla E)^{\perp}(\varphi)=\nabla E(\varphi)-(\nabla E(\varphi), \hat{\tau}) \hat{\tau}
$$

where $(\cdot, \cdot)$ denotes the standard inner product.

To evolve a string in time, at each point on the curve $\varphi$ we consider

$$
\varphi_{t}=-(\nabla E)^{\perp}(\varphi)
$$

which we call the general string dynamics. Indeed, the string method has been analyzed with a dynamical system view in [2]. In practice, to use a suitable form of (2.2) in numerical computation, a particular parametrization of the string can be chosen. Introducing $\alpha$ as the parameter for a suitable parametrization, $\varphi(\alpha, t)$ then gives the instantaneous position of the string at time $t$ with parameter $\alpha$. One simple example of the parametrization is the equal arc-length parametrization for which the following condition holds:

$$
\left(\left|\varphi_{\alpha}\right|\right)_{\alpha}=0
$$

To impose the parametrization, a Lagrange multiplier is introduced, leading to the system

$$
\left\{\begin{array}{l}
\varphi_{t}=-(\nabla E)^{\perp}(\varphi)+\lambda \hat{\tau} \\
\left(\left|\varphi_{\alpha}\right|\right)_{\alpha}=0
\end{array}\right.
$$

which we call the canonical string dynamics here with $\hat{\tau}=\varphi_{\alpha} /\left|\varphi_{\alpha}\right|$. The canonical form takes on a special parametrization not imposed in the general string dynamics, and the Lagrange multiplier $\lambda=\lambda(\alpha, t)$ can be determined by the choice of parametrization [5]. Since the tangential term does not affect the normal velocity of the curve, the re-parametrization of the curve does not change the evolution of the curve. As a result, a simple interpolation step is suggested in [5] to realize the contribution from the term $\lambda \hat{\tau}$ in the numerical algorithm.

It was later noticed that one of the computational complexity in the general or canonical string dynamics lies in the calculation of the projected force. In particular, care must be taken when computing the tangent vector around the saddle point to 
ensure the numerical stability. In [6], an improved string dynamics was developed to eliminate the projection:

$$
\left\{\begin{array}{l}
\varphi_{t}=-\nabla E(\varphi)+\bar{\lambda} \hat{\tau} \\
\left(\left|\varphi_{\alpha}\right|\right)_{\alpha}=0
\end{array}\right.
$$

where $\bar{\lambda}$ remains to be a Lagrange multiplier for enforcing the particular parametrization of the string. To distinguish the different variants of the string method while without completely altering the naming convention used in [6], we call the above system (2.5) the improved canonical string dynamics. Equation (2.5) is in fact equivalent to $(2.4)$ with $\bar{\lambda}=\lambda+(\nabla E(\varphi), \hat{\tau})$.

For the numerical implementation, [6] proposed the use of the simplified string method based on a time splitting scheme. The discretized string is composed of a number of images $\left\{\varphi_{i}(t), i=0,1, \ldots, N\right\}$. A two-step splitting procedure is used in the simplified string method. First, the discrete images on the string are driven by the potential force,

$$
\left(\varphi_{i}\right)_{t}=-\nabla E\left(\varphi_{i}\right)
$$

which is the general string dynamics mentioned above. Then, a parametrization step is applied to redistribute the images along the string. For instance, in the canonical case, an equal arc-length parametrization is enforced. We refer to $[12,15]$ for the latest applications of the string methods.

\section{The constrained string method}

Our objective is to study the string method and some of its variants for computing the MEP and saddle points on a constrained configuration manifold. To deal with the constraints, a natural choice to consider first is the method of Lagrange multipliers.

3.1. Constrained string dynamics. Without loss of generality, we first let

$$
g(\varphi)=0
$$

denote a general constraint. In practice, (3.1) may represent, for example, the constant volume constraint, constant surface area constraint or any other constraints that may arise in applications.

The dynamical equation of the constrained string is then given by

$$
\varphi_{t}=-(\nabla E)^{\perp}(\varphi)+\lambda^{T} \nabla g(\varphi)+\bar{\lambda} \hat{\tau},
$$

where, as before, $\bar{\lambda}$ is the Lagrange multiplier determined by the parametrization, and the new parameter $\lambda$ is the Lagrange multiplier for the constraint (3.1) whose dimension matches with that of the constraint function $g(\varphi)$.

To derive the explicit expression of the Lagrange multiplier $\lambda$, we start by taking the time derivative of $g(\varphi)$ to obtain

$$
\frac{d}{d t} g(\varphi)=\nabla g \varphi_{t}=\left(-(\nabla E)^{\perp}(\varphi)+\lambda^{T} \nabla g(\varphi)+\bar{\lambda} \hat{\tau}\right) \nabla g .
$$

Taking $\alpha$ as the internal parametrization of the string $\varphi$, we have $\hat{\tau}=\varphi_{\alpha} /\left|\varphi_{\alpha}\right|$. Due to the constraint $g(\varphi)=0$ for any $t$ and $\alpha$, we also have

$$
\frac{d}{d t} g(\varphi)=0, \quad \frac{d}{d \alpha} g(\varphi)=0 .
$$


We then obtain $\nabla g(\varphi) \varphi_{\alpha}=0$ and $\nabla g(\varphi) \hat{\tau}=0$. Thus,

$$
(\nabla g \otimes \nabla g) \lambda=\nabla g(\varphi)(\nabla E)^{\perp}(\varphi)-\bar{\lambda} \nabla g(\varphi) \hat{\tau}=\nabla g(\varphi)(\nabla E)^{\perp}(\varphi) .
$$

Here, the symbol $\otimes$ denotes the tensor product having entries $\left(\nabla g_{i} \cdot \nabla g_{j}\right)$ with $\left\{g_{i}\right\}$ being the components of $g(\varphi)$. If $g(\varphi)$ has a single component, then $\nabla g \otimes \nabla g=|\nabla g|^{2}$.

With the equal arc-length parametrization, the constrained canonical string dynamics is then

$$
\left\{\begin{array}{l}
\varphi_{t}=-(\nabla E)^{\perp}(\varphi)+\lambda^{T} \nabla g(\varphi)+\bar{\lambda} \hat{\tau} \\
(\nabla g \otimes \nabla g) \lambda=\nabla g(\nabla E)^{\perp} \\
\left(\left|\varphi_{\alpha}\right|\right)_{\alpha}=0 .
\end{array}\right.
$$

Furthermore, we have the improved constrained canonical string dynamics:

$$
\left\{\begin{array}{l}
\varphi_{t}=-\nabla E(\varphi)+\lambda^{T} \nabla g(\varphi)+\tilde{\lambda} \hat{\tau} \\
(\nabla g \otimes \nabla g) \lambda=\nabla g \nabla E \\
\left(\left|\varphi_{\alpha}\right|\right)_{\alpha}=0
\end{array}\right.
$$

Consequently, the projection step can also be eliminated as well.

We note that in the second equation of (3.3) or (3.4), $\lambda$ is independent of the choice of parametrization $\bar{\lambda}$ or $\tilde{\lambda}$. Thus, in numerical computation, the simplified constrained string method can be efficiently implemented by adopting the simplified string method with a time-splitting scheme.

3.2. Energy dissipation law. As shown before, changing the parametrization of the string does not affect the evolution of the curve so that instead of using systems (3.3) or (3.4), it is more convenient for us to work with the constrained general string dynamics as follows:

$$
\left\{\begin{array}{l}
\varphi_{t}=-(\nabla E)^{\perp}(\varphi)+\lambda^{T} \nabla g(\varphi) \\
(\nabla g \otimes \nabla g) \lambda=\nabla g(\nabla E)^{\perp}
\end{array}\right.
$$

Here, the string parametrization is enforced via the gradient system (3.5).

In [2], Cameron, Kohn and Vanden-Eijnden analyzed the general string method to identify MEPs on a given energy landscape. They pointed out that the string evolution does not necessarily converge to a MEP if the MEPs are not isolated. The same scenario can happen to problems with constraints. To avoid such a situation, we adopt similar conditions in the convergence theorem in [2], properly modified for the constrained setting, to assume that

1. both the potential energy $E$ and the constraint $g$ are twice continuously differentiable;

2. $E$ has isolated nondegenerate constrained critical points; and

3. the constrained sublevel sets $S(C)=\{x: E(x) \leq C$ and $g(x)=0\}$ are compact for any point $x$ of the initial path.

We also assume that the constrained MEPs are isolated to ensure the convergence of the string evolution.

Before deriving the energy dissipation law for the constrained general string method, we first recall it for the general string method without any constraint. The string evolution is given by

$$
\varphi_{t}=-(\nabla E)^{\perp}(\varphi)
$$


and followed by the parametrization. The energy dissipation law can be obtained by taking the time derivative of $E(\varphi)$,

$$
\frac{d}{d t} E=\nabla E \varphi_{t}=-\nabla E(\nabla E)^{\perp}(\varphi)=-\left|(\nabla E)^{\perp}(\varphi)\right|^{2} .
$$

Similarly, for the constrained general string dynamics, we have

Proposition 3.1. The system (3.5) of the constrained general string dynamics preserves the constraint and ensures the energy dissipation law.

Proof. First, we take the time derivative of $g=g(\varphi)$. Using the second equation of (3.5), we have

$$
\frac{d}{d t} g=\nabla g\left(-(\nabla E)^{\perp}(\varphi)+\lambda^{T} \nabla g(\varphi)\right)=0,
$$

so that for every point $\varphi$ on the string, the constraint is satisfied. Furthermore, since

$$
\lambda^{T}(\nabla E, \hat{\tau}) \hat{\tau} \nabla g(\varphi)=\frac{\lambda^{T}}{\left|\varphi_{\alpha}\right|}\left(\nabla E, \frac{\varphi_{\alpha}}{\left|\varphi_{\alpha}\right|}\right) \nabla g(\varphi) \varphi_{\alpha}=0
$$

we have

$$
\begin{aligned}
\frac{d}{d t} E & =\nabla E\left(-(\nabla E)^{\perp}(\varphi)+\lambda^{T} \nabla g(\varphi)\right) \\
& =-\left|(\nabla E)^{\perp}(\varphi)\right|^{2}+\nabla g(\varphi) \lambda^{T}(\nabla E)^{\perp}(\varphi)+\lambda^{T}(\nabla E, \hat{\tau}) \hat{\tau} \nabla g(\varphi) \\
& =-\left|(\nabla E)^{\perp}(\varphi)-\lambda^{T} \nabla g(\varphi)\right|^{2}=-\left|\varphi_{t}\right|^{2} .
\end{aligned}
$$

Thus, both the constraint and energy dissipation law are satisfied.

The above result implies in particular that the maximum of the energy $E$ over the string is non-increasing which provides a form of stability bound, even though the point-wise energy may not always decrease.

\section{Discretization of the constrained string method}

4.1. Time-discretized constrained general string dynamics. Here, we consider the time discretization of the constrained string method. We show in particular that the properly defined scheme can ensure the energy dissipation law while preserving the constraint. These features are more important than the accuracy of the time discretization as our main concern is the constrained MEP calculation which is the asymptotic limit of the constrained general string dynamics as $t \rightarrow \infty$. Let us choose a fully implicit scheme for the system (3.5) as an example:

$$
\left\{\begin{array}{l}
\frac{\varphi^{n+1}-\varphi^{n}}{h}=-\nabla E^{\perp}\left(\varphi^{n+1}, \varphi^{n}\right)+\lambda^{T} \nabla g\left(\varphi^{n+1}, \varphi^{n}\right), \\
\nabla g\left(\varphi^{n+1}, \varphi^{n}\right) \otimes \nabla g\left(\varphi^{n+1}, \varphi^{n}\right) \lambda=\nabla g\left(\varphi^{n+1}, \varphi^{n}\right) \nabla E^{\perp}\left(\varphi^{n+1}, \varphi^{n}\right),
\end{array}\right.
$$

where $h$ is the time step and $\nabla E\left(\varphi^{n+1}, \varphi^{n}\right)$ and $\nabla g\left(\varphi^{n+1}, \varphi^{n}\right)$ satisfy the conditions

$$
\left\{\begin{array}{l}
E\left(\varphi^{n+1}\right)-E\left(\varphi^{n}\right)=\nabla E\left(\varphi^{n+1}, \varphi^{n}\right)\left(\varphi^{n+1}-\varphi^{n}\right) \\
g\left(\varphi^{n+1}\right)-g\left(\varphi^{n}\right)=\nabla g\left(\varphi^{n+1}, \varphi^{n}\right)\left(\varphi^{n+1}-\varphi^{n}\right)
\end{array}\right.
$$

If $\varphi$ is a real scalar variable, the conditions in (4.2) are satisfied by the standard finite differences. For vector-valued $\varphi$ or $\varphi$ defined in a Banach space, it is still possible 
to have (4.2) satisfied for many forms of the energy functional and the constraints as shown in the later examples. We now have

Proposition 4.1. The system (4.1) for the constrained general string dynamics preserves the constraint and ensures the discrete energy dissipation law if the conditions (4.2) are satisfied.

Proof. The conservation of constraint is straightforward,

$$
\begin{aligned}
g\left(\varphi^{n+1}\right)-g\left(\varphi^{n}\right) & =\nabla g\left(\varphi^{n+1}, \varphi^{n}\right) \cdot\left(-\nabla E^{\perp}\left(\varphi^{n+1}, \varphi^{n}\right)+\lambda^{T} \nabla g\left(\varphi^{n+1}, \varphi^{n}\right)\right) h \\
& =0 .
\end{aligned}
$$

Furthermore, we have

$$
\begin{aligned}
E\left(\varphi^{n+1}\right)-E\left(\varphi^{n}\right) & =\nabla E\left(\varphi^{n+1}, \varphi^{n}\right)\left(\varphi^{n+1}-\varphi^{n}\right) \\
& =\nabla E\left(\varphi^{n+1}, \varphi^{n}\right)\left(-\nabla E^{\perp}\left(\varphi^{n+1}, \varphi^{n}\right)+\lambda^{T} \nabla g\left(\varphi^{n+1}, \varphi^{n}\right)\right) h \\
& =-\left|\nabla E^{\perp}\left(\varphi^{n+1}, \varphi^{n}\right)-\lambda^{T} \nabla g\left(\varphi^{n+1}, \varphi^{n}\right)\right|^{2} h \\
& =-\left|\varphi_{n+1}-\varphi^{n}\right|^{2} / h,
\end{aligned}
$$

which is the discrete analog of the energy dissipation law.

4.2. Some illustrative examples. Motivated by our studies of nucleation problems in solid materials and the deformation of vesicle membranes using the diffuse interface methods $[4,16,17]$, we take an energy functional and constraints written in terms of the phase field variables as an example.

Let $\varphi$ be a phase field variable defined in a cubic domain $\Omega$, satisfying periodic boundary condition. Let $\epsilon$ be the interfacial width parameter which is often small. We then consider the following three functionals

$$
\begin{aligned}
& A(\varphi)=\int_{\Omega} \varphi d x \\
& B(\varphi)=\int_{\Omega}\left[\frac{\epsilon}{2}\left|\nabla_{x} \varphi\right|^{2}+\frac{1}{4 \epsilon}\left(\varphi^{2}-1\right)^{2}\right] d x, \\
& C(\varphi)=\int_{\Omega}\left[\epsilon\left(\Delta_{x} \varphi+\frac{1}{\epsilon^{2}}\left(1-\varphi^{2}\right) \varphi\right)^{2}\right] d x .
\end{aligned}
$$

For a typical phase field function $\varphi=\varphi(x)$, the functional $A=A(\varphi)$ can be used to approximate the difference of the volumes on the two sides of $\Gamma$, the zero level surface of $\varphi$. Meanwhile, for $\varphi$ satisfying a certain ansatz assumption, $B=B(\varphi)$ and $C=C(\varphi)$ converge to, up to constant multiplicative factors, the surface area and the elastic bending energy of $\Gamma$ respectively as $\epsilon \rightarrow 0[3,14]$.

In the diffuse interface modeling of binary phase transition problems, we often encounter the problem of computing for the MEP and/or saddle point of $B(\varphi)$ subject to a given value of $A=A(\varphi)$, while in the study of lipid bilayer vesicle deformations, we may consider the transition between different deformed states by computing the MEP of $C=C(\varphi)$ subject to both given values of $A=A(\varphi)$ and $B=B(\varphi)$ [4]. We now illustrate the time discretization of constrained general string method for these two examples. Let

$$
\eta(\varphi)=-\epsilon \Delta_{x} \varphi+\frac{1}{\epsilon}\left(\varphi^{2}-1\right) \varphi
$$


for any phase field function $\varphi=\varphi(x)$ and for any pair of functions $\varphi_{1}, \varphi_{2}$, let

$$
\begin{aligned}
\kappa\left(\varphi_{1}, \varphi_{2}\right) & =-\frac{\epsilon}{2} \Delta_{x}\left(\varphi_{1}+\varphi_{2}\right)+\frac{1}{4 \epsilon}\left(\varphi_{1}^{2}+\varphi_{2}^{2}-2\right)\left(\varphi_{1}+\varphi_{2}\right), \\
\sigma\left(\varphi_{1}, \varphi_{2}\right) & =\frac{1}{\epsilon^{2}}\left(\varphi_{1}^{2}+\varphi_{1} \varphi_{2}+\varphi_{2}^{2}-1\right), \\
\gamma\left(\varphi_{1}, \varphi_{2}\right) & \left.=\sigma\left(\varphi_{1}, \varphi_{2}\right)\left[\eta\left(\varphi_{1}\right)+\eta\left(\varphi_{2}\right)\right]-\Delta_{x}\left[\eta\left(\varphi_{1}\right)+\eta\left(\varphi_{2}\right)\right]\right) .
\end{aligned}
$$

Direct calculation shows that

$$
\begin{aligned}
& B\left(\varphi_{1}\right)-B\left(\varphi_{2}\right)=\int_{\Omega}\left(\varphi_{1}-\varphi_{2}\right) \kappa\left(\varphi_{1}, \varphi_{2}\right) d x, \\
& C\left(\varphi_{1}\right)-C\left(\varphi_{2}\right)=\int_{\Omega}\left(\varphi_{1}-\varphi_{2}\right) \gamma\left(\varphi_{1}, \varphi_{2}\right) d x,
\end{aligned}
$$

which can be used to verify (4.2).

Then, the time-discrete constrained string method for computing the saddle point of $B=B(\varphi)$ subject to the constraint $A(\varphi)=\alpha$ is given by

$$
\left\{\begin{array}{l}
\frac{\varphi^{n+1}-\varphi^{n}}{h}=-\kappa\left(\varphi^{n+1}, \varphi^{n}\right)^{\perp}+\lambda, \\
\int \kappa\left(\varphi^{n+1}, \varphi^{n}\right)^{\perp} d x=\lambda|\Omega| .
\end{array}\right.
$$

Similarly, the time-discrete constrained string method for computing the saddle point of $C=C(\varphi)$ subject to the constraints $A(\varphi)=\alpha$ and $B(\varphi)=\beta$ is given by

$$
\left\{\begin{array}{l}
\frac{\varphi^{n+1}-\varphi^{n}}{h}=-\gamma\left(\varphi^{n+1}, \varphi^{n}\right)^{\perp}+\lambda_{1}+\lambda_{2} \kappa\left(\varphi^{n+1}, \varphi^{n}\right), \\
\int \gamma\left(\varphi^{n+1}, \varphi^{n}\right)^{\perp} d x=\lambda_{1}|\Omega|+\lambda_{2} \int \kappa\left(\varphi^{n+1}, \varphi^{n}\right) d x, \\
\int \gamma\left(\varphi^{n+1}, \varphi^{n}\right)^{\perp} \kappa\left(\varphi^{n+1}, \varphi^{n}\right) d x=\lambda_{1} \int \kappa\left(\varphi^{n+1}, \varphi^{n}\right) d x+\lambda_{2} \int \kappa\left(\varphi^{n+1}, \varphi^{n}\right)^{2} d x .
\end{array}\right.
$$

For both examples, we know from earlier discussion that the discrete energy dissipation laws are satisfied and the constraint(s) are automatically preserved.

In general, implicit schemes can assure energy dissipation while satisfying the constraints and parametrization. Large time steps can often be taken if the time accuracy is not of particular concern. While their implementation appears to be more involved, it is possible to design either Newton-like or optimization-based iterative schemes for their numerical solution. Explicit predictor-corrector type methods can also be used so that sufficient number of corrections would provide good approximations to implicit schemes. In practice, it is not always necessary to implement an implicit scheme as simple explicit schemes and high order time (and space) discretizations may also work well. For instance, it has been shown in [6] that a 4 -th order $\mathrm{R}-\mathrm{K}$ time-integrator can provide effective implementation of the string method when combined with cubic spline interpolation for the string re-parametrization. Such an approach should also be applicable to the constrained case. The numerical examples given later also illustrate how a simple explicit integrator can be implemented. 


\section{Algorithm implementation and numerical simulations}

We now give details on how to implement the constrained canonical string method effectively.

5.1. Algorithm of constrained canonical string method. First, the string is discretized by a number of points, i.e., $\left\{\varphi_{i}(t), i=1, \ldots, N\right\}$, and we may have many different choices for the parametrization. In the string method, after the evolution of the string, a linear or cubic interpolation is used to implement the parametrization. In the simplest case, the equal arc-length parametrization could be enforced in the canonical string method. For the constrained canonical string method, we take advantage of the parametrization and implement the numerical algorithm by following the approach proposed by E, Ren, and Vanden-Eijnden [6] which is outlined below.

Step 1: Evolution of the string: let $\varphi_{i}^{n}, i=0, \ldots, N$ be the positions of the images after $n$ iterations, and for illustration, let us use the explicit Euler scheme; the Lagrange multiplier is then calculated by

$$
\left(\nabla g\left(\varphi_{i}^{n}\right) \otimes \nabla g\left(\varphi_{i}^{n}\right)\right) \lambda_{i}^{n+1}=\nabla g\left(\varphi_{i}^{n}\right) \nabla E\left(\varphi_{i}^{n}\right) .
$$

The new set of images, $\varphi^{\prime}$, satisfies the equation:

$$
\frac{\varphi_{i}^{\prime}-\varphi_{i}^{n}}{h}=-\nabla E\left(\varphi_{i}^{n}\right)+\lambda_{i}^{n+1} \cdot \nabla g\left(\varphi_{i}^{n}\right) .
$$

Step 2: Parametrization of the string by the equal arc-length.

(1) Compute the arc length by the current images,

$$
s_{0}=0, \quad s_{i}=s_{i-1}+\left|\varphi_{i}^{\prime}-\varphi_{i-1}^{\prime}\right|, \quad i=1,2, \ldots, N .
$$

The grid points $\left\{\alpha_{i}^{\prime}\right\}$ are then obtained by $\left\{\alpha_{i}^{\prime}=s_{i} / s_{N}\right\}$.

(2) Apply the linear/cubic interpolation of $\left\{\varphi_{i}^{\prime}\right\}$ to get the new images $\left\{\varphi_{i}^{n+1}\right\}$ at the grid points $\left\{\alpha_{i}=i / N\right\}$.

(3) With $\left\{\varphi_{i}^{n+1}\right\}$ known, go back to Step 1 and iterate until convergence.

5.2. A finite dimensional example. We choose a $3 \mathrm{D}$ constrained problem to illustrate the developed method. Consider the potential energy $E=E(x, y, z)$ as

$$
E(x, y, z)=\left(x^{2}-1\right)^{2}+y^{2}+2 z^{2},
$$

with the constraint

$$
g(x, y, z)=x^{2}+y^{2}+z^{2}-1=0 .
$$

The potential energy $E=E(x, y, z)$ has two minima at $a=(1,0,0)$ and $b=(-1,0,0)$ respectively. Without the constraint, the exact MEP connecting these two minima should be the straight line between $a$ and $b$ (see figure 5.1(left)), and the saddle point is $(0,0,0)$. Taking into account the constraint $g=0$, the exact constrained MEPs connecting two minima are changed to the upper and lower branches of the unit circle in the $(x, y)$ plane, and saddle points are changed to $(0, \pm 1,0)$.

In our calculation, the string is discretized into $N+1$ points $\left\{\left(x_{i}, y_{i}\right)\right\}_{i=0}^{N}$. We used $N$ that ranges from 20 to 200 in the experiments. An explicit scheme is applied for the string evolution, and linear interpolation is used to redistribute the discrete points at each time step according to the equal arc length. We take very small time 

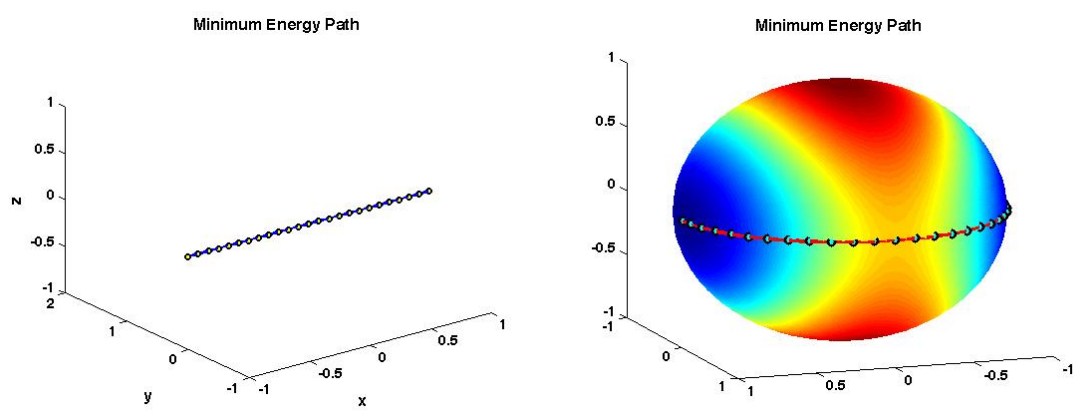

FIG. 5.1. The exact MEP (left) and the calculated constrained MEP (right). The energy landscape on the sphere is highlighted.

steps to ensure the numerical stability. In figure 5.1 (right), the calculated constrained MEP is plotted by a red curve along the equator, and the energy surface on the sphere is highlighted to offer an intuitive understanding.

To show the convergence as we increase the numbers of images, $N$, we compute the error of the converged string defined by $\operatorname{error}(N)=\max _{i}\left|\sqrt{x_{i}^{2}+y_{i}^{2}}-1\right|$. The error decreases linearly vesus $N$, as shown in figure 5.2, due to the use of linear interpolation. The accuracy can be improved by using high order methods such as the cubic spline interpolation presented in [6].

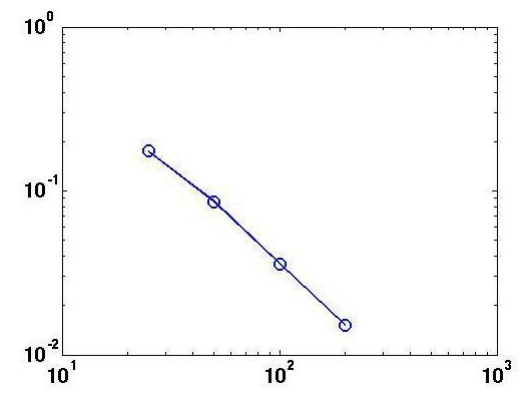

FIG. 5.2. $\operatorname{error}(N)$ of the calculated $M E P$ vs $N$.

\section{Alternative approaches to enforce the constraint}

6.1. Calculation of saddle point by penalty formulation. An alternative way to enforce the constraint is to use a direct penalty term in the improved string dynamics, that is, we may consider a modified total energy functional of the form

$$
E_{M}(\varphi)=E(\varphi)+\frac{M}{2} g(\varphi)^{2}
$$

with $M>0$ being the penalty constant.

The improved string dynamics with the penalty formulation would then become

$$
\varphi_{t}=-\nabla E(\varphi)-M g(\varphi) \nabla g(\varphi)
$$

It is well known that finding a critical point of the energy function subject to the constraint is not equivalent to finding a critical point of the modified energy with 
the penalty as $M \rightarrow \infty$. The saddle-point solution calculated by the penalty method may not be the correct one. However, we will use an example to show that the string method with the penalty formulation could avoid this issue and find the correct saddle point, and the more general case can be derived in the same way.

Consider the $3 \mathrm{D}$ example in section 5.2 , and the modified energy with a penalty term by (5.1) is given by

$$
E(x, y, z)=\left(x^{2}-1\right)^{2}+y^{2}+2 z^{2}+\frac{M}{2}\left(x^{2}+y^{2}+z^{2}-1\right)^{2} .
$$

The critical point satisfies the Euler-Lagrange equation:

$$
\left\{\begin{array}{l}
4 x\left(x^{2}-1\right)+2 M x\left(x^{2}+y^{2}+z^{2}-1\right)=0, \\
2 y+2 M y\left(x^{2}+y^{2}+z^{2}-1\right)=0, \\
4 z+2 M z\left(x^{2}+y^{2}+z^{2}-1\right)=0 .
\end{array}\right.
$$

It is easy to see, as $M \rightarrow \infty$, that

$$
\left(x^{2}+y^{2}+z^{2}-1\right)\left(\begin{array}{l}
x \\
y \\
z
\end{array}\right)=0
$$

So, one saddle-point solution is $(0,0,0)$, which has a lower energy than the other saddle points $(0, \pm 1,0)$, but it does not satisfy the constraint. Thus, using directly the penalty formulation to calculate the saddle point may lead to a wrong solution. More generally, the Euler-Lagrange equation given by the penalty formulation is

$$
\nabla E\left(x_{M}\right)+M g\left(x_{M}\right) \nabla g\left(x_{M}\right)=0 .
$$

One possible situation where the solution $x^{*}$ of the above equation could be a wrong solution is the case when it satisfies both $\nabla E\left(x^{*}\right)=0$ and $\nabla g\left(x^{*}\right)=0$ but $g\left(x^{*}\right) \neq 0$. There are other possibilities that the penalty formulation fails to find the saddle point, such as when $x_{M}$ diverges or $\nabla E\left(x_{M}\right)$ becomes unbounded as $M \rightarrow \infty$.

The string method with the penalty formulation can overcome this flaw of the penalty method. For instance, in the 3D example, if two ends of the string converge to the local minima, every point on the string will satisfy the constraint when the string converges to the MEP. Then the correct saddle-point solution is identified by the point with the highest energy on the MEP. Otherwise, all points on the string will shrink to a single point $(0,0,0)$, which can be circumvented by using the initial string where two ends lie in the two basins of attraction of the minima. Numerical experiments for the 3D example correctly captured this phenomenon.

6.2. Enforce the constraint by augmented Lagrangian method. A direct solution of (6.2) makes the algorithmic implementation much simpler than the system (3.5), but it requires us to choose a large enough $M$ to make sure that $M g(\varphi)$ converges to the corresponding Lagrange multiplier. In order to avoid the stiffness and ill-conditioning associated with a large penalty constant, an augmented Lagrangian approach $[8,9]$ can be utilized which is based on modifying the total energy by

$$
E_{A L M}(\varphi)=E(\varphi)+\lambda^{T} g(\varphi)+\frac{M}{2} g(\varphi)^{2}
$$

where the penalty constant $M>0$ becomes a fixed parameter.

The augmented Lagrangian method is implemented as follows: 
Step 1: For $j \geq 0$, assume that $\lambda_{j}$ and $\varphi_{j}$ are known, we let $\varphi_{j+1}(0)=\varphi_{j}$ and apply the simplified string method to evolve the string associated with the energy $E(\varphi)+\lambda_{j}^{T} g(\varphi)+\frac{M}{2} g(\varphi)^{2}$ to get $\varphi_{j+1}$.

Step 2: Once the solution $\varphi_{j+1}$ is obtained, we update the multipler by

$$
\lambda_{j+1}=\lambda_{j}+M g\left(\varphi_{j+1}\right) \nabla g\left(\varphi_{j+1}\right) .
$$

Then we take $\lambda_{j+1}$ and $\varphi_{j+1}$ back to Step 1 and iterate until convergence.

The augmented Lagrangian method has the advantage that the penalty constant need not be very large and assures the convergence of the Lagrange multiplier and thus the satisfaction of the constraint. In the numerical implementation, the initial estimate of $\lambda^{T}$ and the initial string can be obtained from the direct penalty method with a suitable penalty constant.

As an example, we consider the energy functional $B=B(\varphi)$ subject to the constraint functional $A=A(\varphi)$, as defined in section 4.2. For small $\epsilon$, this leads to a diffuse interface description of the nucleation process governed by the interfacial energy for a concentration field subject to volume conservation [1]. To add a driving force, we modify $B=B(\varphi)$ slightly as

$$
B(\varphi)=\int_{\Omega}\left\{\frac{\epsilon}{2}\left|\nabla_{x} \varphi\right|^{2}+\frac{1}{4 \epsilon}\left[\left(\varphi^{2}-1\right)^{2}-\left(\varphi_{0}^{2}-1\right)^{2}-4\left(\varphi-\varphi_{0}\right)\left(\varphi_{0}^{3}-\varphi_{0}\right)\right]\right\} d x
$$

with $\varphi_{0}$ being the value of the concentration in the homogeneous state.

In the numerical computation, we choose $\Omega$ as a two dimensional square $(-1,1)^{2}$ and set $\epsilon=1 / 64$. We fix one end of the string to be the initial state representing a uniform concentration with $\varphi_{0}=-0.94$ in $\Omega$, while allowing the other end to move but generally within the energy well of the ground state or equilibrium solution. We use 31 points to discretize the string and the Fourier spectral method with a $256 \times 256$ grid for computing the concentration profiles, i.e., points on the string. Using the augmented Lagrangian formulation of the constrained string method, we are led to the MEP given in figure 6.1 (left). The saddle point (middle) and the equilibrium solution (right) are also given in figure 6.1. The variations of the saddle point and equilibrium solutions are mostly concentrated near the origin, so that only the portions of the solutions in the center of the square are plotted to offer a better view. While both the saddle point and the equilibrium solution show some qualitative similarity with their circular contours, we have found dramatically different saddle points and equlibrium solutions when the elasticity effect is introduced, see [19] for more detailed discussions where additional examples on the application of the augmented Lagrangian approach can also be found.
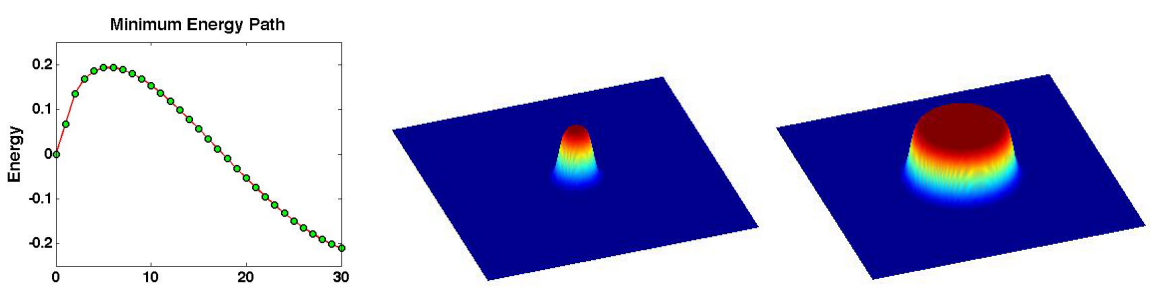

FIG. 6.1. the MEP (left), the saddle point (middle) and the equilibrium solution (right) 


\section{Conclusion}

In this paper, a constrained string method is developed to solve the minimum energy path and/or saddle-point problems with general constraints. By taking advantage of a Lagrange multiplier formulation for the constraint, the constrained string method can be readily implemented without changing the intrinsic description of the string method proposed in [5]. Furthermore, some analysis is provided for the constrained string method, including the demonstration of the energy dissipation law while satisfying the constraints. It is also noticed that with the Lagrange multiplier formulation, the equations to determine the Lagrange multipliers are in fact independent of the choice of parametrization for both the constrained canonical string method and the simplified or improved versions. In addition, some time discretization schemes for the constrained string method are analyzed and nice approximation features are presented. To offer more understanding and perhaps alternatives, other approaches to enforce the constraints are also considered, such as the penalty formulation and augmented Lagrangian method. The latter provides a simpler algorithmic implementation. More applications of the constrained string method are currently underway in the study of nucleation and phase transition processes in materials and biology.

Acknowledgment. The authors would like to thank Profs. Weinan E, Weiqing Ren and Eric Vanden-Eijnden for their discussion of the string method. They also thank Prof. Robert Kohn for bringing [2] to their attention.

\section{REFERENCES}

[1] J. Cahn and J. Hilliard, Free energy of a nonuniform system. III. nucleation in a two-component incompressible fluid, J. Chem. Phys., 31, 688-699, 1959.

[2] M. Cameron, R. Kohn and E. Vanden-Eijnden, The string method as a dynamical system, preprint, 2009.

[3] Q. Du, C. Liu, R. Ryham and X. Wang, A phase field formulation of the Willmore problem, Nonlinearity, 18, 1249-1267, 2005.

[4] Q. Du, C. Liu and X. Wang, A phase field approach in the numerical study of the elastic bending energy for vesicle membranes, J. Comput. Phys., 198, 450-468, 2004.

[5] W. E, W. Ren and E Vanden-Eijnden, String method for the study of rare events, Physical Review B, 66, 052301, 2002.

[6] W. E, W. Ren and E Vanden-Eijnden, Simplified and improved string method for computing the minimum energy paths in barrier-crossing events, J. Chem. Phys., 126, 164103, 2007.

[7] L.C. Evans, Partial Differential Equations, AMS, 1998.

[8] R. Glowinski and P.L. Tallec, Augmented Lagrangian and Operator-Splitting Methods in Nonlinear Mechanics, SIAM, 1989.

[9] M. Hestenes, Multipler and gradient methods, Journal of Optimization Theory and Applications, 4, 303-320, 1969.

[10] G. Henkelman and H. Jónsson, A dimer method for finding saddle points on high dimensional potential surfaces using only first derivatives, J. Chem. Phys., 111, 7010-7022, 1999.

[11] G. Henkelman, B. Uberuaga and H. Jónsson, A climbing image nudged elastic band method for finding saddle points and minimum energy paths, J. Chem. Phys., 113, 9901-9904, 2000.

[12] C. Qiu, T.Z. Qian and W. Ren, Application of the string method to the study of critical nuclei in capillary condensation, J. Chem. Phys., 129, 154711, 2008.

[13] P. Rabinowitz, Minimax Methods in Critical Point Theory With Applications to Differential Equations, AMS, Provindence, 1986.

[14] M. Röger and R. Schätzle, On a modified conjecture of DeGiorgi, Mathematische Zeitschrift, 254, 675-714, 2006.

[15] M. Venturoli, E. Vanden-Eijnden and G. Ciccotti, Kinetics of phase transitions in two dimensional Ising models studied with the string method, J. Math. Chem., 45, 188-222, 2009.

[16] L. Zhang, L.Q. Chen and Q. Du, Morphology of critical nuclei in solid state phase transformations, Phys. Rev. Lett., 98(25), 265703, 2007.

[17] L. Zhang, L.Q. Chen and Q. Du, Diffuse-interface description of strain-dominated morphology of critical nuclei in phase transformations, Acta Materialia, 56, 3568-3576, 2008. 
[18] L. Zhang, L.Q. Chen and Q. Du, Mathematical and numerical aspects of phase-field approach to critical morphology in solids, J. Sci. Comput., 37, 89-102, 2008.

[19] L. Zhang, L.Q. Chen and Q. Du, Simultaneous prediction of morphologies of a critical nucleus and an equilibrium precipitate in solids, Commun. Comput. Phys., 7, 674-682, 2010. 